\title{
Determination of the optimal time for radioiodine therapy in anaplastic thyroid carcinoma using the adenovirus- mediated transfer of sodium iodide symporter gene
}

\author{
YOUNG SO ${ }^{1}$, YONG JIN LEE ${ }^{2}$, WON WOO LEE ${ }^{3,5}$ and JUNE-KEY CHUNG ${ }^{4,6}$
}

${ }^{1}$ Department of Nuclear Medicine, Konkuk University School of Medicine, Chungju; ${ }^{2}$ Molecular Imaging Research Center, Korea Institute of Radiological and Medical Sciences, Seoul; ${ }^{3}$ Seoul National University College of Medicine, Seoul National University Bundang Hospital, Seongnam; ${ }^{4}$ Seoul National University Hospital, Seoul; ${ }^{5}$ Institute of Radiation Medicine, Medical Research Center, Seoul National University, Seoul;

${ }^{6}$ Cancer Research Institute, Seoul National University College of Medicine, Seoul, Republic of Korea

Received October 12, 2012; Accepted December 28, 2012

DOI: $10.3892 /$ or.2013.2277

\begin{abstract}
Gene therapy using human sodium iodide symporter (hNIS) and radioiodine has been considered promising in a variety of gene therapy trials. However, the optimal timing of radioiodine application following hNIS gene transfer remains unknown. The present study aimed to investigate the serial expression of hNIS following adenovirus-mediated hNIS gene transfer into anaplastic thyroid carcinoma (ARO) to determine the optimal timing of radioiodine application. Recombinant adenovirus encoding the hNIS gene (rAd-hNIS) was generated using a homologous recombination reaction. The iodine uptake of rAd-hNIS-transfected ARO cells gradually increased until 120 min post- ${ }^{125}$ I application but the fold increase, reflecting the relative uptake of rAd-hNIS-transfected compared to non-transfected ARO cells, reached plateau at 60 min post- ${ }^{125} \mathrm{I}$ application. For the in vivo analysis, rAd-hNIS was injected intratumorally into ARO cell xenografts in the thighs of nude mice $(\mathrm{n}=12)$. Two, 3, 4 and 6 days after rAd-hNIS injection, $\gamma$-scintigraphic images were obtained $60 \mathrm{~min}$ following injection of $5.5 \mathrm{MBq}$ of ${ }^{131} \mathrm{I}$ intraperitoneally. Treated/non-treated (T/NT) xenograft count ratios were the highest at day 2 post-rAd-hNIS injection (2.85 \pm 0.61$)$, and gradually decreased thereafter $(2.54 \pm 0.65,2.31 \pm 0.42$ and $2.18 \pm 0.90$ at days 3,4 and 6 post-rAd-hNIS injection, respectively). Real-time poly-
\end{abstract}

Correspondence to: Dr Won Woo Lee, Seoul National University College of Medicine, Seoul National University Bundang Hospital, 173-82 Gumi-ro, Bundang-gu, Seongnam, Gyeonggi-do 463-707, Republic of Korea

E-mail:wwlee@snu.ac.kr

Dr June-Key Chung, Seoul National University College of Medicine, Seoul National University Hospital, 101 Daehang-ro, Jongno-gu, Seoul 110-744, Republic of Korea

E-mail: jkchung@plaza.snu.ac.kr

Key words: sodium iodide symporter, adenovirus, radioiodine, anaplastic thyroid carcinoma merase chain reaction (RT-PCR) and immunohistochemical staining demonstrated that hNIS expression was the highest at day 2 following rAd-hNIS injection. In conclusion, the optimal timing for radioiodine administration is day 2 after adenovirus-mediated hNIS gene transfer into anaplastic thyroid carcinoma.

\section{Introduction}

Radioiodine plays a key role in the diagnosis and treatment of differentiated thyroid cancers, and is used to diagnose recurrent and metastatic disease and to treat differentiated thyroid cancers (1-3). The success of radioiodine for the treatment of differentiated thyroid cancers lies in the iodine-concentrating ability of the cancer, whereas most non-iodine-concentrating thyroid cancers result in treatment failure. However, if the iodine-concentrating function of thyroid cancers is restored, then radioiodine therapy would become feasible $(4,5)$.

Sodium iodide symporter (NIS) is an integral membrane glycoprotein that mediates the active transport of iodine into thyroid follicular cells, the first step of thyroid hormone synthesis (6-8). The ability of the thyroid to concentrate iodine via NIS, provides the bases for thyroid diagnostic scintigraphic imaging using radioiodine and radioiodine therapy in hyperthyroidism and thyroid cancer.

Since NIS was cloned and characterized in $1996(6,7)$, a number of studies have been conducted on NIS (9-13). At present, NIS is considered a novel therapeutic gene $(4,14)$, since it offers a way of restoring the therapeutic effect of ${ }^{131} \mathrm{I}$ in anaplastic and poorly differentiated thyroid carcinomas $(15,16)$. Thus, coupling the delivery of the NIS gene into tumor cells by ${ }^{131} \mathrm{I}$ administration may open new avenues of radionuclide gene therapy. However, the majority of previous studies have used ex vivo NIS gene transfer to produce engineered cancer cell lines stably expressing NIS (11-14). In such a condition of constitutive NIS expression, there is no restriction of radioiodine application with regard to the timing. However, such situations do not apply to gene therapy in clinic, in which the expression of NIS is limited in terms of time and place 
since the NIS gene should be exogenously delivered to the target tumor. Radioiodine application should be matched with NIS expression accordingly. In this regard, we believe that the in vivo transfection of NIS gene is a more reasonable means of emphasizing the application of radioiodine gene therapy in clinical practice.

The aim of this study was to investigate the optimal timing of radioiodine therapy during adenovirus-mediated human sodium iodide symporter (hNIS) gene transfer into anaplastic thyroid cancer (ARO) cells.

\section{Materials and methods}

Cell lines. The human ARO cell line was obtained from the American Type Culture Collection (ATCC; Rockville, MD, USA). ARO cells were grown in RPMI-1640 medium supplemented with $10 \%$ fetal bovine serum (FBS), $100 \mathrm{IU} / \mathrm{ml}$ penicillin and $100 \mu \mathrm{g} / \mathrm{ml}$ streptomycin at $37^{\circ} \mathrm{C}$ in $5 \% \mathrm{CO}_{2}$, and when $40-80 \%$ confluent, they were transfected with recombinant adenoviral vector.

Human embryonic kidney (HEK) 293 cells were also obtained from ATCC, and adenovirus-transformed HEK 293 cells were maintained in Dulbecco's modified Eagle's medium (DMEM; Gibco-BRL, Grand Island, NY, USA), containing $10 \% \mathrm{FBS}, 2 \mathrm{mM}$ L-glutamine, $100 \mathrm{IU} / \mathrm{ml}$ penicillin and $100 \mu \mathrm{g} / \mathrm{ml}$ streptomycin.

Cloning of a recombinant adenoviral vector for hNIS gene transfer. Recombinant adenoviral vector encoding hNIS (rAd-hNIS) was produced using a homologous recombination reaction. hNIS cDNA was kindly provided by Dr Sissy M. Jhiang of the Ohio State University (7) and was cloned using the AdEasy ${ }^{\mathrm{TM}}$ system (Qbiogene, Montréal, Canada), which contains a green fluorescent protein (GFP) gene, and uses a homologous recombination of a shuttle vector and a backbone bacteria plasmid. Briefly, the hNIS cDNA gene was first cloned into a shuttle vector, pAdTrack-CMV, and the resultant plasmid was linearized by digestion with restriction endonuclease $P m e I$ and subsequently cotransformed into E. coli. BJ5183 recombinants containing the adenoviral backbone plasmid, pAdEasy-1, were selected for kanamycin resistance, and recombination was confirmed by restriction endonuclease analysis. The linearized recombinant plasmid was then transfected into an adenovirus packaging cell line, HEK 293. Recombinant adenovirusproducing foci were easily confirmed using fluorescence microscopy following HEK 293 transfection by observing GFP expression (17).

Adenovirus-mediated hNIS gene transfer in ARO cells in vitro. ARO cells $\left(2 \times 10^{5}\right)$ were added to each well in 24-well plates, and then incubated for $24 \mathrm{~h}$ in $0.5 \mathrm{ml}$ RPMI media. The cells in each well were then transfected with rAd-hNIS at multiplicities of infection (MOIs) of $0,2.5,5$ or 10 . Fortyeight hours after transfection, $0.1 \mu \mathrm{Ci}(3.7 \mathrm{MBq})$ of ${ }^{125} \mathrm{I}$ in $10 \mu \mathrm{M}$ of cold iodine was applied to each well and incubated for $10,30,60,90$ or $120 \mathrm{~min}$ in quadruplicate. To perform inhibition assays, we inhibited hNIS activity by adding $50 \mathrm{M}$ potassium perchlorate to a separate $10 \mathrm{MOI}$ quadruplicate. Following incubation, wells were washed with cold Hank's balanced salt solution (HBSS) and radioactivities were counted using a $\gamma$-counter. A protein assay was also performed to calculate iodine uptake (pmol) per $\mathrm{mg}$ of protein in each well. The values quoted were the means of experiments performed in quadruplicate.

Adenovirus-mediated hNIS gene transfer in ARO cell xenografts in vivo. Three-week-old male BALB/c nude mice $(n=12)$ were obtained from the Charles River Laboratories (Yokohama, Japan). The experiments were approved by our Institutional Animal Research Committee. Levothyroxine sodium (50-100 $\mu \mathrm{g} / \mathrm{kg} /$ day) was supplemented in drinking water to block thyroid ${ }^{131}$ I uptake.

Fifteen days after $2 \times 10^{6}$ ARO cells were subcutaneously injected in $200 \mu \mathrm{l}$ of sterile phosphate-buffered saline (PBS) into both thighs of 12 nude mice (when ARO cell xenografts had reached $8-10 \mathrm{~mm}$ in diameter), and $1.5 \times 10^{8}$ plaque-forming units (pfu) of rAd-hNIS in $50 \mu \mathrm{l}$ PBS was injected into the ARO cell xenografts in the right thighs $(n=12)(T$, treated tumor). The same amount of normal saline was injected into ARO cell xenografts in the left thighs (NT, non-treated tumor). rAd-hNIS and normal saline were injected into 4 sites within each xenograft using 30-gauge insulin syringes.

Scintigraphic ${ }^{131}$ I images of adenovirus-mediated hNIS gene transformed ARO cell xenografts. Two, 3, 4 or 6 days following intratumoral injection of rAd-hNIS, ${ }^{131}$ I images were captured using a $\gamma$-camera (Sigma 410 Radioisotope Camera, Ohio-Nuclear, Inc., Solon, OH, USA) equipped with a pinhole collimator. Nude mice were anesthetized with an intraperitoneal (i.p.) injection of $53 \mathrm{mg} / \mathrm{kg}$ ketamine and $12 \mathrm{mg} / \mathrm{kg}$ xylazine and placed under the collimator in a prone position. Sixty minutes after an i.p. injection of $5.5 \mathrm{MBq}$ of ${ }^{131} \mathrm{I}, 5-\mathrm{min}$ static images of the 12 mice ( 3 mice per day) were captured. Treated/non-treated (T/NT) count ratios were calculated at 60 min post- ${ }^{131}$ I injection for each mouse. ARO xenografts were excised in all the cases after images were captured and preserved at $-70^{\circ} \mathrm{C}$ until the following experiment.

Detection of hNIS mRNA expression in ARO cell xenografts by real-time polymerase chain reaction $(R T-P C R)$. The following primer pairs were used to detect hNIS mRNA using RT-PCR: 5'-GCT AAG TGG CTT CTG GGT TG-3' (hNIS gene sense primer); 5'-GTA AGC ACA GGC CAG GAA AA-3' (hNIS gene antisense primer). These hNIS gene primer pairs corresponded to the coding regions 941-960 and 1300-1319 and yielded a product of $379 \mathrm{bp}$ for the hNIS gene. For comparison purposes, RT-PCR for the housekeeping gene glyceraldehydes3-phosphate dehydrogenase (GAPDH) was also performed using the primer pairs: 5'-ACC AGG GCT GCT TTT AAC TCT-3' (GAPDH gene sense primer); 5'-GAG TCC TTC CAC GAT ACC AAA G-3' (GAPDH gene antisense primer). The GAPDH gene primer pairs corresponded to the coding regions 130-150 and 576-597 and yielded a 468-bp product. Total RNA was isolated using RNeasy Midi kits (Qiagen Inc., Valencia, CA, USA) and a rotor-stator homogenizer. RT-PCR was performed over 27 amplification cycles using a GeneAmp® PCR System 9700 (Applied Biosystem, Inc., Foster City, CA). The PCR hNIS gene and GAPDH gene fragments were analyzed by $1.5 \%$ agarose/ethidium bromide gel electrophoresis. 


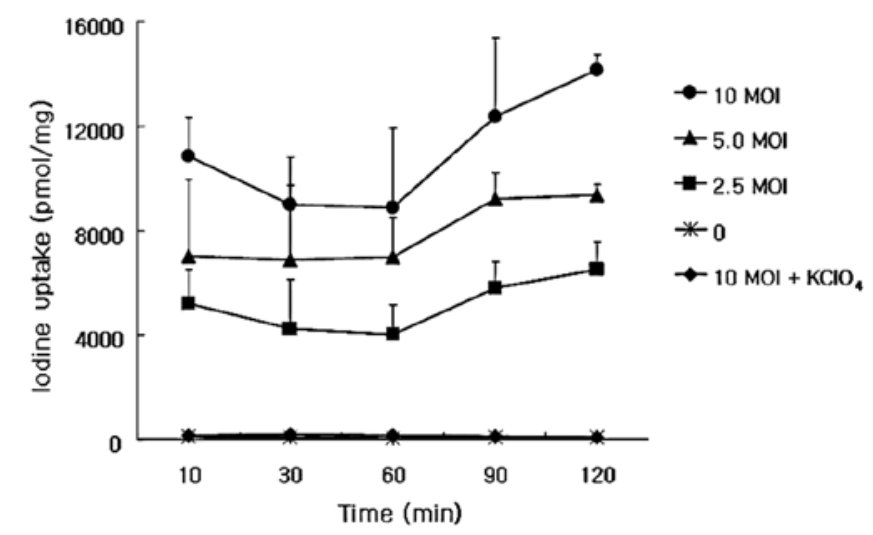

Figure 1. Iodine uptake analysis of adenovirus-mediated hNIS-transfected ARO cells. Iodine uptake increased until 120 min post- ${ }^{131} \mathrm{I}$ administration at viral titers of 2.5, 5.0 and 10 MOIs. Increased iodine uptake was inhibited by potassium perchlorate.

Immunohistochemical staining of excised ARO cell xenografts. Immunohistochemical staining of paraffin-embedded tissue sections derived from ARO cell xenografts was performed using rabbit anti-rat thyroid iodide transporter IgG (TIT11-A, Alpha Diagnostic International, San Antonio, TX, USA). Tissue sections were deparaffinized by three passages in xylene, subjected to a graded series of ethanol washes $(100$, 95 and $90 \%$ ethanol solutions) and then washed in distilled water. Endogenous peroxidase activity was blocked by incubation in $3 \% \mathrm{H}_{2} \mathrm{O}_{2}$ /methanol for $10 \mathrm{~min}$ and sections were washed in Tris-buffered saline and Tween-20 (TBS Tween-20: pH 7.4 \pm 0.05 , Tris $0.005 \mathrm{M}, \mathrm{NaCl} 0.15 \mathrm{M}$, Tween-20 0.05\%). To expose antigens, slides were heated in $0.01 \mathrm{M}$ citrate buffer for $12 \mathrm{~min}$. After cooling to room temperature for $20 \mathrm{~min}$, slides were incubated using primary antibodies (TIT11-A diluted to 1:100) for $60 \mathrm{~min}$, and washed with TBS Tween-20, anti-rabbit secondary antibody (EnVision ${ }^{\mathrm{TM}}+$, K 4003 HRP, Rabbit, DakoCytomation Inc., Glostrup, Denmark) for 40 min. 3,3'-Diaminobenzidine was used as the chromogen. Slides were counterstained with Mayer's hematoxylin and observed under a light microscope.

\section{Results}

In vitro iodine uptake analysis of adenovirus-mediated hNIS gene-transfected ARO cells. The iodine uptake of adenovirus-mediated hNIS gene-transfected ARO cells increased for $120 \mathrm{~min}$ at viral titers of 2.5, 5.0 and $10 \mathrm{MOIs}$, and was completely inhibited when potassium perchlorate was administered (Fig. 1). The fold-increase of iodine uptake by ARO cells transfected with the rAd-hNIS versus non-transfected ARO cells also increased for $120 \mathrm{~min}$, but there was a leap forward $\sim 60$ min post-iodine application (Fig. 2). Thus, after the radioiodine administration, the 60 min time point was selected for scintigraphic imaging studies.

Scintigraphic ${ }^{131}$ I images of adenovirus-mediated hNIS gene-transfected ARO cell xenografts. ARO cell xenografts in the right thighs of nude mice were readily visualized $60 \mathrm{~min}$ after administering ${ }^{131} \mathrm{I}$ on days 2, 3, 4 and 6 following recombinant adenovirus injeciton (Fig. 3). ${ }^{131} \mathrm{I}$ accumulation

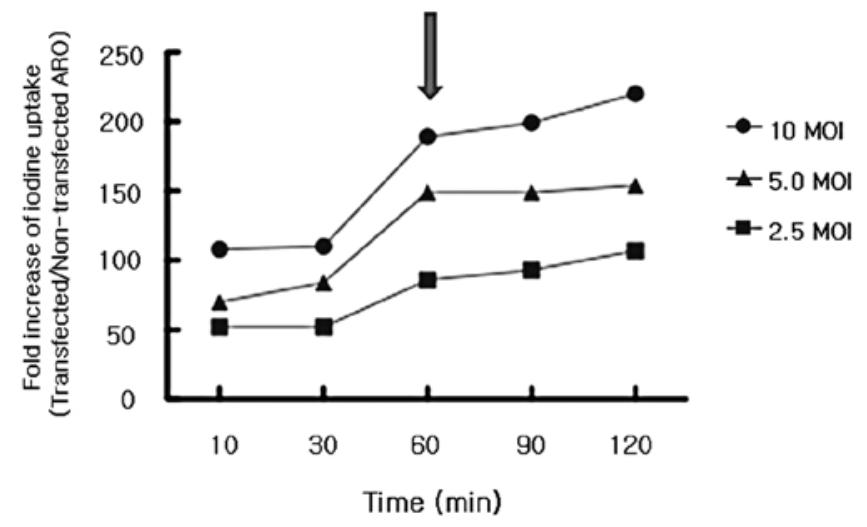

Figure 2. Fold increase of ${ }^{125} \mathrm{I}$ uptake by ARO cells transfected with recombinant adenovirus encoding the hNIS gene (rAd-hNIS) compared with non-transfected ARO cells. There was a leap forward of the relative uptake of iodine $\sim 60$ min post-iodine administration (arrow).

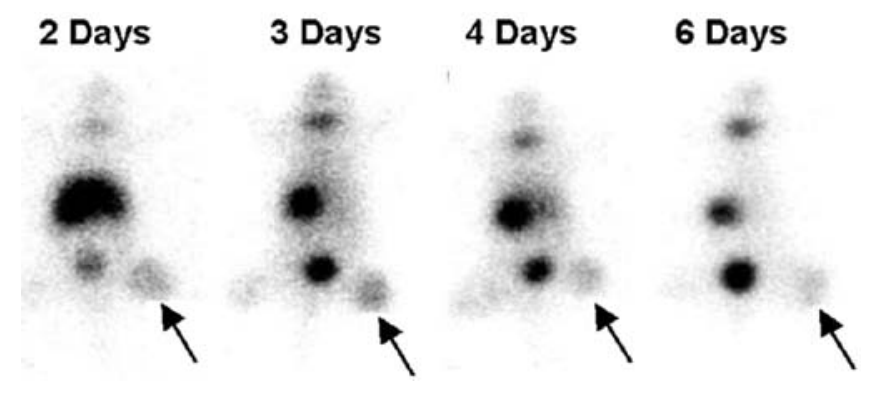

Figure 3. Representative 60-min ${ }^{131}$ I static images of BALB/c nude mice 2, 3,4 and 6 days after the intratumoral injection of recombinant adenovirus encoding hNIS into ARO cell xenografts in right thighs (arrows). Transfected ARO cell xenografts were readily visualized in the day 2 and 3 images, however, ${ }^{131} \mathrm{I}$ uptake using xenografts was gradually reduced thereafter. Images of mice are in prone position.

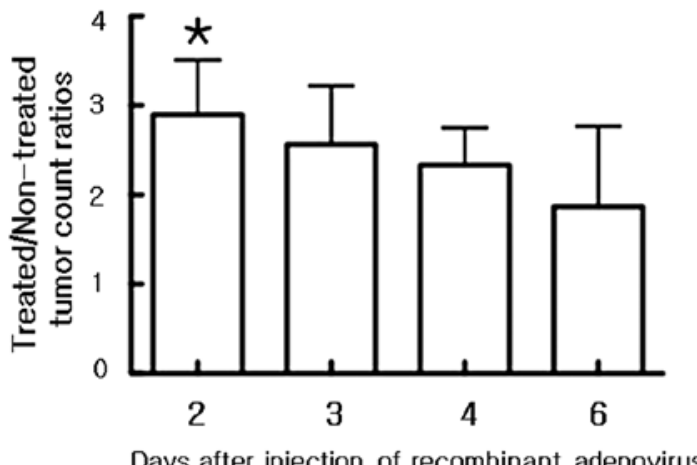

Figure 4. Treated/non-treated (T/NT) tumor count ratios of adenovirus-mediated hNIS-transfected ARO cell xenografts in ${ }^{131} \mathrm{I}$ images captured $60 \mathrm{~min}$ post-injection. Mean count ratios on days 2, 3, 4 and 6 after intratumoral virus injection were $2.85 \pm 0.61,2.54 \pm 0.65,2.31 \pm 0.42$ and $2.18 \pm 0.90$, respectively. ${ }^{*}$ The T/NT ratio at day 2 post-adenovirus injection was the highest of the other time points.

by xenografts was more prominent on the day 2 and 3 images compared to the day 4 and 6 images, which showed a gradual reduction. Mean T/NT count ratios of ARO cell xenografts of ${ }^{131}$ I images on days $2,3,4$ and 6 were $2.85 \pm 0.61,2.54 \pm 0.65$, $2.31 \pm 0.42$ and $2.18 \pm 0.90$, respectively (Fig. 4). 


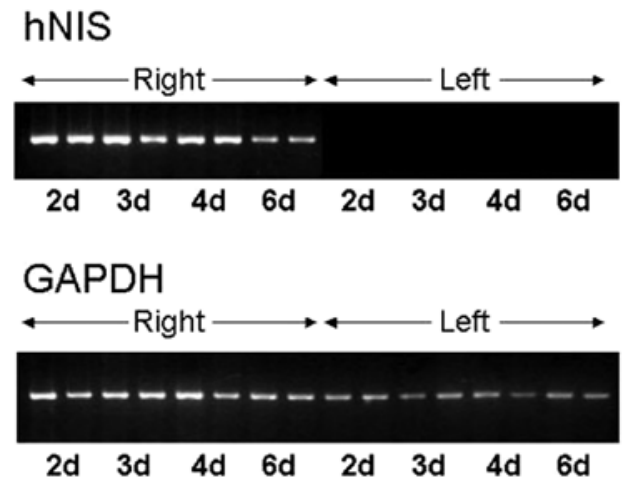

Figure 5. RT-PCR products of hNIS mRNA and GAPDH mRNA from ARO cell xenografts. Amplified 379-bp hNIS dsDNA band intensities on days 2, 3 and 4 post-recombinant adenovirus intratumoral injection were higher compared to those of amplified 468-bp GAPDH dsDNA bands in the same specimens. hNIS band intensities (379 bp) peaked on day 2 and then gradually decreased.

RT-PCR results of adenovirus-mediated hNIS gene-transfected ARO cell xenografts. RT-PCR for hNIS mRNA, which was extracted from adenovirus-transfected ARO cell xenografts excised from the right thighs of nude mice, produced a 379-bp hNIS dsDNA RT-PCR product, whereas RT-PCR for hNIS mRNA extracted from normal saline-injected ARO cell xenografts into left thighs did not yield any product. Amplified 379-bp hNIS dsDNA band intensities at 2, 3 and 4 days post-recombinant adenovirus injection were higher compared to those of amplified 468-bp GAPDH dsDNA bands in the same specimens. Moreover, the band intensities of hNIS mRNA RT-PCR products were the highest on day 2 and then gradually decreased, and on day 6 these intensities were lower compared to those of the GAPDH mRNA RT-PCR products (Fig. 5).

Immunohistochemical staining results of adenovirus-mediated hNIS gene-transfected ARO cell xenografts. Immunohistochemical staining of excised ARO cell xenografts was performed to determine the immunohistochemical localization of hNIS in adenovirus-mediated hNIS gene-transfected ARO cells. hNIS expression was the highest in ARO cell xenograft tissue specimens excised 2 days post-recombinant adenovirus injection, which then gradually decreased. Necrotic areas were most abundant in ARO cell xenografts excised at 6 days post-recombinant adenovirus injection. hNIS expression was predominantly found in the cytoplasmic membranes of adenovirus-mediated hNIS gene-transfected ARO cells (Fig. 6), whereas no hNIS expression was observed in tissue specimens excised from ARO cell xenografts injected intratumorally with normal saline.

\section{Discussion}

In the present study, we successfully transferred the hNIS gene in vivo by intratumorally injecting recombinant adenovirus encoding the hNIS gene (rAd-hNIS), and serially measured transferred hNIS gene expression 2, 3, 4 and 6 days following recombinant virus injection using radioiodine imaging, RT-PCR and immunohistochemistry. The results showed that 2 days post-intratumoral injection is an optimal time for radioiodine therapy using the hNIS gene.

Thyroid cancer has been a target of gene therapy using NIS gene and radioiodine. Shimura et al (15) and Smit et al (16) reported that transfection of the NIS gene into thyroid cancer cells resulted in radioiodine accumulation. However, those authors used cell lines stably expressing the NIS gene. In the present study, we injected recombinant adenovirus encoding hNIS in vivo intratumorally to more accurately reflect perceived clinical applications.

In vivo NIS gene transfer has been investigated in a variety of cancer cells using adenoviral vectors. Boland et al (9) injected ${ }^{131}$ I i.p. 3 days following intratumoral injection of recombinant adenovirus into a human cervical tumor cell xenograft. Cho et al (10) injected ${ }^{125}$ I i.p. $43 \mathrm{~h}$ after recombinant adenovirus intratumoral injection into a human glioma cell xenograft, and Spitzweg et al (18) injected ${ }^{123}$ I i.p. 4 days after recombinant adenovirus intratumoral injection into a human prostate cancer cell xenograft. However, those studies did not mention the optimal timing of radioiodine application since most were performed only at a single time point.

When it comes to the use of adenoviral vector as a vehicle, an initial high expression followed by a subsequent reduction in hNIS expression delivered by adenovirus has been reported in a non-tumor animal model (19). However, a thorough evaluation of adenovirus-mediated hNIS expression in terms of the optimal timing of radioiodine anti-tumor therapy has yet to be conducted.
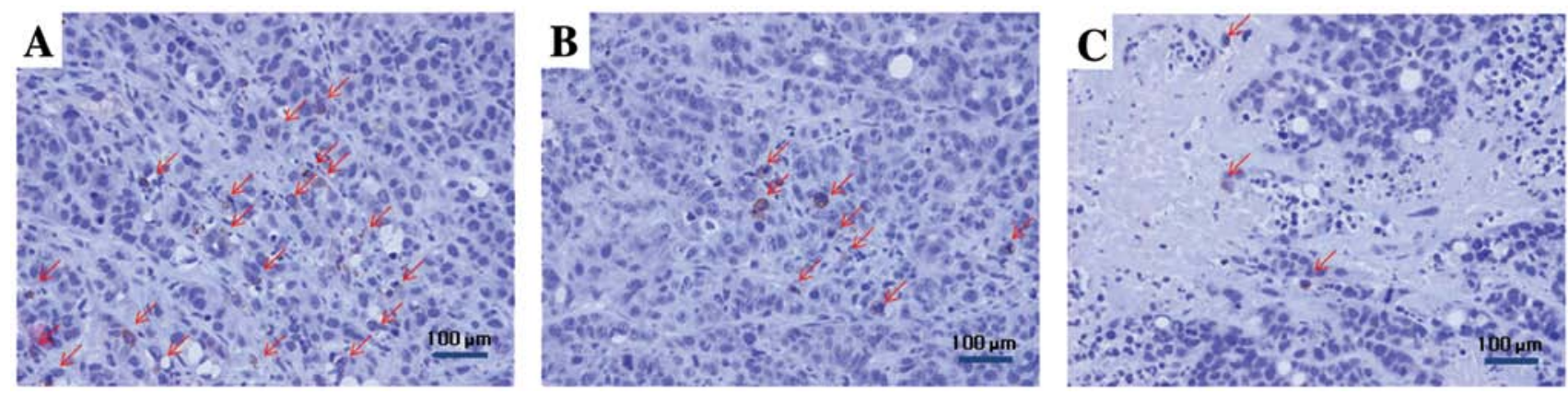

Figure 6. Immunohistochemical localization of hNIS expression in adenovirus-mediated hNIS gene-transfected ARO cell xenografts. hNIS expression was the highest in specimens excised on day 2 (A) after intratumoral recombinant adenovirus injection and decreased on day 3 (B) and was the lowest in specimens excised on day $6(\mathrm{C})$ post-injection (original magnification, $\mathrm{x} 400$ ). Arrows indicate hNIS-expressing tumor cells. 
Gene therapy using hNIS and ${ }^{131} \mathrm{I}$ administration have encountered several obstacles that need to be overcome. One of these obstacles is the rapid washout of delivered radioiodine. In their study, Spitzweg et al calculated that the average biological half-life of ${ }^{131} \mathrm{I}$, which enters a tumor-expressing $\mathrm{hNIS}$, is only $5.6 \mathrm{~h}(18)$. However, the average radioiodine half-life in metastatic thyroid cancer patients responding to radioiodine therapy was reported to be as long as 5.5 days (1). The application of tissue-specific promoters, or cotransfection of the thyroperoxidase gene, or the application of high-energy $\beta$-ray emitting radioisotopes such as ${ }^{188} \mathrm{Re}$, may help resolve this problem $(13,20-22)$. Increased knowledge of NIS and the development of gene therapy techniques should enable the identification of a role for the NIS gene in radionuclide gene therapy in the near future $(4,5)$.

In conclusion, radioiodine uptake was successfully increased in ARO tumors by adenovirus-mediated hNIS gene transfer in vitro and in vivo. The optimal time for radioiodine administration (day 2 post-recombinant adenovirus injection) was determined by serial imaging and analysis. The results obtained during this study suggest the possibility of applying radioiodine therapy in iodine non-concentrating tumors by hNIS gene transfer.

\section{Acknowledgements}

This study was supported in part by a grant of the Korea Healthcare technology R\&D Project, Ministry of Health and Welfare (A111627), and by grants from the National Research Foundation (NRF), Ministry of Education, Science and Technology (MEST), (2012M2B2A9A02029612; Basic Science Research Program, 2012R1A1A2001060; and Global Core Research Center (GCRC) program, 2011-0030680), Republic of Korea.

\section{References}

1. Maxon HR, Thomas SR, Hertzberg VS, Kereiakes JG, Chen IW, Sperling MI and Saenger EL: Relation between effective radiation dose and outcome of radioiodine therapy for thyroid cancer. N Engl J Med 309: 937-941, 1983.

2. DeGroot LJ, Kaplan EL, McCormick M and Straus FH: Natural history, treatment, and course of papillary thyroid carcinoma. J Clin Endocrinol Metab 71: 414-424, 1990.

3. Schlumberger MJ: Papillary and follicular thyroid carcinoma. N Engl J Med 338: 297-306, 1998.

4. Chung JK: Sodium iodide symporter: its role in nuclear medicine. J Nucl Med 43: 1188-1200, 2002.
5. Chung JK, Youn HW, Kang JH, Lee HY and Kang KW: Sodium iodide symporter and the radioiodine treatment of thyroid carcinoma. Nucl Med Mol Imaging 44: 4-14, 2010.

6. Dai G, Levy O and Carrasco N: Cloning and characterization of the thyroid iodide transporter. Nature 379: 458-460, 1996.

7. Smanik PA, Liu Q, Furminger TL, Ryu K, Xing S, Mazzaferri EL and Jhiang SM: Cloning of the human sodium iodide symporter. Biochem Biophys Res Commun 226: 339-345, 1996.

8. Dohan O, De la Vieja A, Paroder V, et al: The sodium/iodide Symporter (NIS): characterization, regulation, and medical significance. Endocr Rev 24: 48-77, 2003.

9. Boland A, Ricard M, Opolon P, et al: Adenovirus-mediated transfer of the thyroid sodium/iodide symporter gene into tumors for a targeted radiotherapy. Cancer Res 60: 3484-3492, 2000.

10. Cho JY, Xing S, Liu X, et al: Expression and activity of human $\mathrm{Na}^{+} / \mathrm{I}^{-}$symporter in human glioma cells by adenovirus-mediated gene delivery. Gene Ther 7: 740-749, 2000.

11. Spitzweg C, O'Connor MK, Bergert ER, Tindall DJ, Young CY and Morris JC: Treatment of prostate cancer by radioiodine therapy after tissue-specific expression of the sodium iodide symporter. Cancer Res 60: 6526-6530, 2000.

12. Cho JY, Shen DH, Yang W, et al: In vivo imaging and radioiodine therapy following sodium iodide symporter gene transfer in animal model of intracerebral gliomas. Gene Ther 9: 1139-1145, 2002.

13. Schipper ML, Weber A, Behe M, et al: Radioiodide treatment after sodium iodide symporter gene transfer is a highly effective therapy in neuroendocrine tumor cells. Cancer Res 63: 1333-1338, 2003.

14. Mandell RB, Mandell LZ and Link CJ Jr: Radioisotope concentrator gene therapy using the sodium/iodide symporter gene. Cancer Res 59: 661-668, 1999.

15. Shimura H, Haraguchi K, Miyazaki A, Endo T and Onaya T: Iodide uptake and experimental ${ }^{131} \mathrm{I}$ therapy in transplanted undifferentiated thyroid cancer cells expressing the $\mathrm{Na}^{+} / \mathrm{I}^{-}$symporter gene. Endocrinology 138: 4493-4496, 1997.

16. Smit JW, Shroder-van der Elst JP, Karperien M, et al: Reestablishment of in vitro and in vivo iodide uptake by transfection of the human sodium iodide symporter (hNIS) in a hNIS defective human thyroid carcinoma cell line. Thyroid 10: 939-943, 2000.

17. He TC, Zhou S, da Costa LT, Yu J, Kinzler KW and Vogelstein B: A simplified system for generating recombinant adenoviruses. Proc Natl Acad Sci USA 95: 2509-2514, 1998.

18. Spitzweg C, Dietz AB, O'Connor MK, Bergert ER, Tindall DJ, Young CY and Morris JC: In vivo sodium iodide symporter gene therapy of prostate cancer. Gene Ther 8: 1524-1531, 2001.

19. Lee WW, Moon DH, Park SY, Jin J, Kim SJ and Lee H: Imaging of adenovirus-mediated expression of human sodium iodide symporter gene by $99 \mathrm{mTcO} 4$ scintigraphy in mice. Nucl Med Biol 31: 31-40, 2004

20. Huang M, Batra RK, Kogai T, et al: Ectopic expression of the thyroperoxidase gene augments radioiodide uptake and retention mediated by the sodium iodide symporter in non-small cell lung cancer. Cancer Fene Ther 8: 612-618, 2001.

21. Zhang L, Sharma S, Zhu LX, et al: Nonradioactive iodide effectively induces apoptosis in genetically modified lung cancer cells. Cancer Res 63: 5065-5072, 2003.

22. Dadachova E, Bouzahzah B, Zuckier LS and Pestell RG: Rhenium-188 as an alternative to Iodine-131 for treatment of breast tumors expressing the sodium/iodide symporter (NIS). Nucl Med Biol 29: 13-18, 2002. 